\title{
THE CATEGORY OF COFINAL TYPES. II ( $\left.{ }^{1}\right)$
}

\author{
BY
}

J. R. ISBELL

Introduction. The paper is concerned almost entirely with directed sets, and for those the structure introduced in Part I [3] reduces to the usual (Tukey [7]) ordering by "power for convergence." Three problems are central: completeness (least upper bounds), standard sets (basically Tukey's $\Delta_{\lambda_{\mu}}$, the set of all subsets of power $<\boldsymbol{K}_{\lambda}$ of a set of power $\boldsymbol{K}_{\mu}$ ), spectra (the spectrum of $D$ is the set of all standard sets $\leqq D$ ).

On completeness, we answer Tukey's questions: does every set of directed sets have a least upper bound, or every finite set a greatest lower bound? The answers are negative. A countable set of directed sets has either 1 or 2 minimal upper bounds, and every upper bound exceeds a minimal one. For $\left\{D_{i}\right\}$ to have a least upper bound that is not the least upper bound of a finite subset, one must have $D_{i} \geqq \omega_{0}$ for some $i$; conversely, if some $D_{i} \geqq \omega_{0}$, the least upper bound exists (see 2.2, 2.3, 2.4).

There are partial results about least upper bounds of uncountable families, with uncountable standard sets replacing $\omega_{0}$ above; but the necessary conditions and the sufficient conditions are far apart $(2.2,2.12$, 4.15).

For standard sets, we usually (and throughout this paragraph) assume the generalized continuum hypothesis $(\mathrm{H})$. J. Schmidt has characterized [6] (using (H)) many of Tukey's $\Delta_{\lambda_{\mu}}$, including all those for which $\boldsymbol{\aleph}_{\lambda}$ and $\boldsymbol{\aleph}_{\mu}$ are regular. We give a dual $\left({ }^{*}\right)$ of Schmidt's characterization, which holds for the same $\Delta_{\lambda \mu}$ and others, including all for which $\boldsymbol{\aleph}_{\mu}$ is regular. However, some $\Delta_{\lambda,}$ are equivalent to others. The size of this difficulty matters, because we take steps to reduce it. $\Delta_{\lambda_{\mu}}$ is equivalent to $\Delta_{\lambda_{\mu+1}}$ at least if $\boldsymbol{\kappa}_{\lambda}$ is singular and $\mu$ is a limit of $\left\langle\boldsymbol{N}_{\lambda}\right.$ smaller ordinals. Now the dual characterization $\left({ }^{*}\right)$ determines a directed set $\Delta_{\lambda_{\mu}}^{*}$ almost always; precisely, when $\lambda<\mu$ or $\lambda=\mu$ with $\boldsymbol{K}_{\lambda}$ regular. Up to an equivalence, $\Delta_{\lambda_{\mu}}^{*}$ is the set of all sets of power $\left\langle\boldsymbol{N}_{\lambda}\right.$ of ordinal numbers bounded below $\omega_{\mu}$; and the characteristic property is that this is $\left({ }^{* *}\right)$ a least directed set of power $\boldsymbol{N}_{\mu}$, the powers of whose bounded subsets are precisely the cardinal numbers $\left\langle\boldsymbol{\aleph}_{\lambda} .{ }^{* *}\right)$ is not really $\left({ }^{*}\right)$, but it is similar, and does characterize a class of directed sets all of which are equivalent to $\Delta_{\lambda_{\mu}}^{*}$. (See \$4.)

Received by the editors April 13, 1964.

( ) Supported by the National Science Foundation. 
A systematic attempt is made to free the results on standard sets from dependence on $(\mathrm{H})$, which succeeds completely for $\lambda<\mu \leqq \omega_{0}$.

The point spectrum $Q(D)$ of a directed set $D$ is the set of all regular alephs $\aleph_{\alpha}$ such that $D \geqq \omega_{\alpha}$. The standard sets $\Delta_{\lambda \mu}^{*}$ with $\lambda<\mu$ yield a band spectrum, on which we have no global results, though the ordering of the standard sets themselves is determined (if $(\mathrm{H}))$. ( $\left(\Delta_{\lambda \mu}^{*}\right.$ exceeds "most" of the $\Delta_{\alpha \beta}^{*}$ for which $\lambda \leqq \alpha \leqq \beta \leqq \mu$ and at most two others.) The necessary conditions found here for a set $Q$ of cardinals to be a point spectrum are sufficient if $Q$ has power $<\boldsymbol{\aleph}_{2}$, if $(\mathrm{H})$. It is not known whether new conditions apply near $\aleph_{\omega_{2}}$. There is a new condition applying near $\rho$-numbers. ( $\rho$-numbers [4] are so large that they may not exist. These results are in §3.)

Additional results, depending on $(\mathrm{H}) . Q(D)$ determines the smallest power of a cofinal subset of $D$. If $D$ has no well-ordered cofinal subset, $Q(D)$ has more than one element. The set of all integer-valued functions on a set of $\aleph_{\alpha}$ elements, for each $\omega_{\alpha}$ not equivalent to $\omega_{0}$, is equivalent to $\Delta_{0_{\alpha+1}}$. Without $(\mathrm{H})$ : every infinite set, of power $m$, has ultrafilters equivalent to the standard set $\Delta_{0 \beta}$, where $\aleph_{\beta}=2^{m}$.

I am indebted to the referee for fifty constructive suggestions.

1. Preliminaries. This paper is more nearly a sequel to the work of Tukey [7] and Schmidt [6] than a sequel to Part I [3]. More fully, the problems arise naturally from [7], the development of methods is begun in [6], and [3] provides a point of view. Knowledge of any one of those papers should be sufficient background for reading this paper.

It is convenient to reverse the convention of [3] and speak of sets directed upward; $y$ is a successor of $x$ if $y \geqq x$. Concerning degenerate cases we agree that a directed set must be nonempty. Sets having a last element are admitted; their cofinal type will be called zero type.

The fundamental relation between directed sets, denoted $D \geqq E$ in [7], is defined equivalently in [6] and [3] in different language. $D \geqq E$, or $D$ is cofinally finer than $E$, or $E$ is a quotient of $D$, provided there exists a function $f: D \rightarrow E$ of the type called cofinal in [6], convergent in [3] and here. Three characterizations proved in [6] and two mentioned in [3] overlap and are stated in 1.1 below. A remarkable phenomenon stressed by Schmidt [6] (implicit in Tukey [7]): $D \geqq E$ if and only if there exists a function $g: E \rightarrow D$ satisfying conditions indicated in 1.1; Schmidt calls these terminal functions, but we shall call them Tukey functions.

We must consider cofinal, bounded, and unbounded subsets; noncofinal sets, which we call thin; and sets whose complements are thin, which are called perfinal in [6], total in [3] and here.

1.1. For directed sets $D, E$, a function $f: D \rightarrow E$ is convergent if and only if for every convergent function $g$ from $E$ to a topological space, gf converges 
to the same limit; or, the inverse image of every total set (in E) is total; or, the inverse image of every thin set is thin; or, the image of every cofinal set (in $D$ ) is cofinal. Tukey functions $g: E \rightarrow D$ are characterized as follows: the inverse image of every bounded set is bounded; or, the image of every unbounded set is unbounded.

Some remarks concerning the categorical point of view of [3] and Schmidt's "duality" between convergent functions and Tukey functions. Directed sets (not of zero type) and convergent functions form a category $\mathscr{C}_{1}$. Identifying all mappings that have the same domain and the same range, we have a quotient category $\mathscr{C}_{2}$ of directed cofinal types. No more structure remains beyond the fact that for directed sets $D, E$, the number of mappings from $D$ to $E$ may be $1(D \geqq E)$ or 0 . Again, directed sets and Tukey functions form a category $\mathscr{C}_{3}$. Collapsing $\mathscr{C}_{3}$ like $\mathscr{C}_{1}$, we have a quotient category $\mathscr{C}_{4}$. Note that all $\mathscr{C}_{i}$ have the same objects. The duality is that the identity function between the objects of $\mathscr{C}_{2}$ and $\mathscr{C}_{4}$ induces a categorical anti-isomorphism or duality.

Another relation between $\mathscr{C}_{2}$ and $\mathscr{C}_{4}$ is of some interest (though of no use, up to the present time). Let us define a category $\mathscr{C}_{5}$ as follows. An object of $\mathscr{C}_{5}$ consists of a set $X$ and an ideal $I$ of subsets of $X$; a mapping $f$ from $\left(X_{1}, I_{1}\right)$ to $\left(X_{2}, I_{2}\right)$ is a function from $X_{1}$ to $X_{2}$ such that inverse images of members of $I_{2}$ are members of $I_{1}$. Then both $\mathscr{L}_{1}$ and $\mathscr{C}_{3}$ can be embedded in $\mathscr{C}_{5}$. For $\mathscr{C}_{1}$, associate to $D$ the pair $\left({ }^{2}\right)(D, I)$, where $I$ is the ideal of thin sets; for $\mathscr{C}_{3}$, use the ideal of bounded sets. If $\mathscr{C}_{5}$ is collapsed as before, we have a category $\mathscr{C}_{6}$ containing copies of $\mathscr{C}_{2}$ and $\mathscr{C}_{4}$. $\mathscr{C}_{6}$ has some attractive properties, such as completeness [4] (this is virtually the same as being a complete lattice, if the notion $D \geqq E$ is suitably extended). From [6], it is easy to identify the intersection of $\mathscr{C}_{2}$ and $\mathscr{C}_{4}$ in $\mathscr{C}_{6}$ as the set of minimal elements of $\mathscr{L}_{2}$, corresponding to well-ordered sets.

Schmidt shows that every directed set $D$ is equivalent $(D \geqq E \geqq D)$ to a directed set $E$ that is complete, i.e. every bounded subset of $E$ has a least upper bound. Equivalently, every nonempty subset has a greatest lower bound. A companion result:

1.2. Every isotone function from a directed set $D$ onto a cofinal subset of a directed set $E$ is convergent. If $E$ is complete and $D \geqq E$, then there exists an isotone convergent function $f: D \rightarrow E$.

Proof. If $f: D \rightarrow E$ is isotone and $S$ is cofinal in $D$, then $f(S)$ is cofinal in $f(D)$, hence also in $E$ when $f(D)$ is cofinal in $E$. Supposing that $g: D \rightarrow E$ is convergent and $E$ is complete, define $f: D \rightarrow E$ as follows: $f(x)$ is the greatest lower bound of the set of values of $g$ on successors of $x$. Evidently $f$ is isotone. For each $y$ in $E$, consider the thin set $T$ of nonsuccessors of $y$.

(2) This construction gives an embedding in the standard sense for functors, not required to be one-to-one on objects; an artificial modification would give a one-to-one embedding. 
$g^{-1}(T)$ is thin; so for some $x$ in $D$, no successor of $x$ is in $g^{-1}(T)$, and $f(x)$ $\geqq y$. Thus $f(D)$ is cofinal, and the proof is complete.

We conclude the preliminaries with some remarks on cardinal properties, cardinal sets, and cardinal quotients. Schmidt defines [6] two numbers which we shall call the lower character and upper character of a directed set $D$. The lower character $l(D)$ is the smallest cardinal belonging to an unbounded subset of $D$; the upper character $u(D)$ is the smallest cardinal of a cofinal subset. This leaves $l(D)$ undefined if $D$ has zero type. Schmidt suggests leaving it undefined; no extension of the definition permits extension of the following basic result.

1.3 (Schmidt). If $E$ is a nonzero quotient of $D$, then $l(D) \leqq l(E) \leqq u(E)$ $\leqq u(D)$. In particular, $u(E) \geqq l(E)$, and the characters are invariants of cofinal type.

For every ordinal number $\alpha$, let $W(\alpha)$ denote the set of ordinals less than $\alpha$, directed in the usual way (by inclusion). (Note also that $W(\alpha)$ is logically identical with $\alpha$; but the different notations for the individual $\alpha$ and the set $W(\alpha)$ will be convenient.) $K(\alpha)$ denotes the cofinal type of $W(\alpha)$. There is of course a smallest $W(\beta)$ in $K(\alpha) ; \beta=\operatorname{ch}(\alpha)$ is called the character of $\alpha$, and is a regular cardinal. A cardinal set will mean a set order-isomorphic with some $W(\beta)$, where $\beta$ is a regular aleph; their cofinal types are the cardinal types. A cardinal quotient of a directed set $D$ is defined as a regular aleph that is a quotient of $D$.

Sets of cardinal type are characterized $[6]$ by $l(D)=u(D)$. More fully, the following conditions on a directed set $D$, not of zero type, are equivalent:

(a) $D$ has cardinal type.

(b) $D$ is a quotient of a well-ordered set.

(c) $D$ has the same upper and lower character.

(d) $D$ has a well-ordered cofinal subset.

Proof. See [6], or argue as follows. Trivially (a) $\Longrightarrow$ (b) and (d) $\Longrightarrow(a)$; by $1.3,(b) \Longrightarrow(c)$. From (c), one has a cofinal subset of power $m$, and every smaller set is bounded; so (d) follows easily.

The cardinal sets show that every regular aleph is both the upper character and the lower character of some directed set. Conversely, every lower character $l(D)$ is regular [6]. However, every infinite cardinal $m$ is the upper character of the stack [7] of all finite subsets of a set of power $m$.

The relation $l(D) \leqq u(D)$ can be improved to

1.4. $l(D) \leqq \operatorname{ch}(u(D))$.

This will follow from 1.6 and 1.7. Assuming the generalized continuum hypothesis, Schmidt's examples [6] demonstrate the converse: if $l=\operatorname{ch}(l)$ $\leqq \operatorname{ch}(m)$ then $l$ and $m$ are the characters of some directed set.

1.5. Let $D$ be a directed set and $m$ an infinite cardinal. For $D \geqq W(m)$, a sufficient condition is that $D$ contains $m$ thin sets whose union is cofinal 
but such that no union of fewer than $m$ of them is cofinal. This condition is necessary if $m$ is regular. In that case an equivalent condition is that $D$ has $m$ elements no $m$ of which are bounded.

Proof. Given the indicated thin sets $S_{\alpha}$, indexed by elements $\alpha$ of $W(m)$, and whose union $E$ is cofinal in $D$, define $f: E \rightarrow W(m)$ to take each $x \in E$ to the first $\alpha$ such that $x \in S_{\alpha}$. The inverse image of any initial segment, thus of any thin set, is thin. Hence $E \geqq W(m)$, and $D$ is equivalent to $E$. Conversely, if $m$ is regular and $f: D \rightarrow W(m)$ convergent, the sets $f^{-1}(\alpha)$ form such a family of thin sets. The last assertion is obvious from the properties of Tukey functions.

The second condition in 1.5 is necessary for singular $m$ also, if repetition of elements is allowed. However, the first condition is not necessary $\left(\omega_{0} \geqq W\left(\boldsymbol{N}_{\omega}\right)\right)$, and from Schmidt's examples [6], the second condition is not sufficient.

1.6. The lower character of a directed set $D$ not of zero type is the smallest cardinal quotient of $D$.

Proof. If $S$ is an unbounded subset of minimum power and $S$ admits a one-to-one correspondence with $W(m)$, then one can associate bounds to the initial segements to get $m$ elements no $m$ of which are bounded. Since lower characters are regular, $m$ is a cardinal quotient of $D$. No smaller set $W(n)$ admits a Tukey function to $D$, for the image cannot be unbounded.

1.7. A directed set $D$ of upper character $m$ admits a mapping to $W(m)$; $\operatorname{ch}(m)$ is a cardinal quotient.

Proof. Let $S$ be a cofinal set of minimum power $m$. The sets $P(s)$ of predecessors of $s \in S$ are thin, and so are unions of fewer than $m$ of them, but not the union of all of them. Hence 1.7, and 1.4.

2. Products. Tukey showed [7] that the Cartesian product of two directed sets, ordered coordinatewise, is their least upper bound, or in categorical terminology, their direct product. We shall mention free sums also, i.e. greatest lower bounds. Note that the directed free sum (which need not exist) is quite different from the free sum in the category [3] of all cofinal types (a disjoint union).

The Cartesian product $\times D_{\alpha}$ of a family of directed sets $D_{\alpha}$, ordered coordinatewise, will be called their strong product. It need not represent a categorical product of the types of the $D_{\alpha}$, but it is an invariant of cofinal type. More generally, $D_{\alpha} \geqq E_{\alpha}$ for all $\alpha$ implies $\times D_{\alpha} \geqq X E_{\alpha}$; this is proved in [6]. Apropos of some cases in later proofs, the family of factors can be empty.

A weak product $W$ of a family of directed sets $D_{\alpha}$, none of zero type, is a subset of the strong product constructed as follows. Choose any base points $b_{\alpha} \in D_{\alpha}$, and define $W$ as the set of all $x$ in $\times D_{\alpha}$ such that $x_{\alpha} \geqq b_{\alpha}$ 
for all $\alpha$ and $x_{\alpha}=b_{\alpha}$ for all but finitely many $\alpha$. The type of $W$ depends only on the types of the $D_{\alpha}$; indeed.

2.1. If, for all $\alpha$ in $A, E_{\alpha}$ is a nonzero quotient of $D_{\alpha}$, then any weak product of $\left\{E_{\alpha}: \alpha \in A\right\}$ is a quotient of any weak product of $\left\{D_{\alpha}: \alpha \in A\right\}$.

We need not prove 2.1 separately, for it follows from the identification (2.2) of $W$ as a direct product (not generally of the apparent factors). Let $\Delta_{0}(A)$ denote the stack of all finite subsets of $A$.

2.2. A weak product of $\left\{D_{\alpha}: \alpha \in A\right\}$ is a categorical direct product of $\left\{D_{\alpha}: \alpha \in A\right\}$ and $\Delta_{0}(A)$. Thus it is a direct product of $\left\{D_{\alpha}\right\}$ if some $D_{\alpha}$ has $\Delta_{0}(A)$ as a quotient.

Proof. First, a weak product $W$ with base points $b_{\alpha}$ admits an isotone mapping onto $\Delta(A)$, each $x$ going to the set of all $\alpha$ for which $x_{\alpha} \neq b_{\alpha}$. Evidently $W$ also has convergent coordinate projections to the factors $D_{\alpha}$. Suppose $C$ is a partially ordered set having convergent functions $f_{\alpha}$ : $C \rightarrow D_{\alpha}$ and $f_{0}: C \rightarrow \Delta_{0}(A)$. Define $f: C \rightarrow W$ as follows. The $\alpha$ th coordinate of $f(c)$ is $f_{\alpha}(c)$ provided $\alpha \in f_{0}(c)$ and $f_{\alpha}(c) \geqq b_{\alpha}$, but otherwise it is $b_{\alpha}$. For any total set $T$ in $W, T$ contains the set of all successors of some $x \in W$. Sufficient conditions for $f(c) \geqq x$ are that $f_{0}(c)$ contains the set of indices $\alpha$ such that $x_{\alpha} \neq b_{\alpha}$ and, for these indices, $f_{\alpha}(c) \geqq x_{\alpha}$. This finite set of conditions is all satisfied on a total set; so $f$ is convergent.

2.3. Theorem. If a countable set of directed sets $D_{i}$ has a direct product $P$, then $P$ is equivalent either to the product of a finite set of factors $D_{i}$ or to the weak product of all $D_{i}$.

Proof. If $P$ is a quotient of some finite partial product $Q$, then (by directness) $P$ is equivalent to $Q$. Suppose this is not the case. Replace $P$ by an equivalent complete directed set $R$. A weak product $W$ of all $D_{i}$ $(i=1,2, \ldots)$ is a union of subsets $Q_{n}$ which are directed sets each equivalent to the product of $D_{1}, \cdots, D_{n}$. (Define $Q_{n}$ by setting each coordinate at $i>n$ equal to $b_{i}$.) $R$, as a direct product, must be a quotient of $W$. By 1.2 there is a convergent isotone function $f: W \rightarrow R$. Since $R$ is not a quotient of $Q_{n}, f\left(Q_{n}\right)$ is thin for each $n$; but the union of all $f\left(Q_{n}\right)$ is cofinal in $R$. By $1.5, R \geqq \omega_{0}$. By 2.2 , then, $R \geqq W ; P$ is equivalent to $W$.

Tukey asked [7] whether every set of directed sets has a least upper bound (direct product). To see that the answer is negative one need only find a countable family for which finite products and the weak product fail to be a direct product. The family of all $\omega_{n}\left(1 \leqq n<\omega_{0}\right)$ will do; clearly the finite products are too small, and since the strong product has lower character $\aleph_{1}$ it admits no convergent function to the weak product.

The same example can be treated differently, so as to answer also Tukey's question whether two directed sets must have a free sum. For the set of all $\omega_{n}$, the strong product has lower character $\boldsymbol{\aleph}_{1}$ and the weak 
product has upper character $\boldsymbol{\kappa}_{\omega}$. A directed set $D$ "in between," i.e. a quotient of both products which has all $\omega_{n}$ as quotients, would have these numbers as its characters, contrary to 1.4. No such set exists, no such product, no such sum. In Schmidt's language, the upper semilattice of directed types is not a lattice.

2.4. Theorem. For any countable set of directed sets $D_{i}$, each directed set having all $D_{i}$ as quotients has either their strong product or their weak product as a quotient.

Proof. Consider any such directed set $C$. By 2.2 and 1.6, $C$ has the weak product of all $D_{i}$ as a quotient unless $l(C)>\boldsymbol{\aleph}_{0}$. In that case, consider Tukey functions $g_{i}: D_{i} \rightarrow C$. For each $x=\left(x_{j}\right)$ in the strong product, define $g(x)$ to be some upper bound for the countable set $\left\{g_{j}\left(x_{j}\right)\right\}$. An unbounded set $S$ in the strong product has unbounded $j$ th coordinates for some $j$, so that $g(S)$ is unbounded and $g$ is a Tukey function.

For uncountably many factors our results $(2.5,2.12,4.15)$ are much weaker. There are obvious modifications of the strong and weak products. For any ordinal $\lambda$ and any set of (at least $\boldsymbol{K}_{\lambda}$ ) factors $D_{\alpha}$, none of zero type, we define a $\lambda$-product of $\left\{D_{\alpha}\right\}$ by choosing base points $b_{\alpha} \in D_{\alpha}$ and taking all $x$ in $\times D_{\alpha}$ such that $x_{\alpha} \geqq b_{\alpha}$ and $x_{\alpha}=b_{\alpha}$ with fewer than $\aleph_{\lambda}$ exceptions. Thus a 0 -product is a weak product.

2.1 generalizes to $\lambda$-products; one can prove this by treating Tukey functions coordinatewise, first modifying them to preserve base points.

There is only one application of $\lambda$-products $(\lambda>0)$ in this paper, 1 products occurring in the proof of 3.11. But it requires a lemma (2.11), and we need to mention here the associated directed sets $\Delta_{\lambda}(A)$ of all subsets of power $<\boldsymbol{K}_{\lambda}$ of a set $A$. For any $\lambda$-product of factors $D_{\alpha}(\alpha \in A)$, with base points $b_{\alpha}$, there is a projection to $\Delta_{\lambda}(A)$, taking each $x$ to the set of all $a$ for which $x_{\alpha} \neq b_{\alpha}$. Further, if $A$ has power $m$, the upper character of $\Delta_{\lambda+1}(A)$ is at most $m^{\aleph_{\lambda}}$.

In general we define a subdirect product of a family of directed sets $D_{\alpha}$ of nonzero types as a directed subset $C$ of the strong product $\times D_{\alpha}$ which projects upon a cofinal subset of each factor $D_{\alpha}$. As the coordinate projections are isotone, $C \geqq D_{\alpha}$ for all $\alpha$.

The notion of subdirect product is not an invariant of cofinal type.

Proof. Let $\left\{D_{\alpha}\right\}$ be any infinite family of directed sets, none of zero type. Let $n$ be a very large regular cardinal. Let $E_{\alpha}$ be a disjoint union of $D_{\alpha}$ and $W(n)$, ordered so that all of $W(n)$ precedes all of $D_{\alpha}$. Then $D_{\alpha}$ is a cofinal subset of $E_{\alpha}$, and the types are the same. In more $\times E_{\alpha}$, consider the set of all elements all but finitely many of whose coordinates are in $W(n)$. Evidently this is a subdirect product, and its upper character is not less than $n$. But one can choose $n$ so large that no subdirect product of $\left\{D_{\alpha}\right\}$ has this upper character. 
Despite this drawback, subdirect products are essential for much of what follows. The reason:

2.5. Let $\left\{D_{\alpha}\right\}$ be a family of complete directed sets of nonzero types. Any directed set $C$ which has all $D_{\alpha}$ as quotients has some subdirect product of $\left\{D_{\alpha}\right\}$ as a quotient. Hence $\left\{D_{\alpha}\right\}$ has a direct product if and only if the subdirect products of $\left\{D_{\alpha}\right\}$ have a free sum, and if there is a direct product, some subdirect product is direct.

Proof. If $C$ has all $D_{a}$ as quotients, there are isotone convergent functions $f_{\alpha}: C \rightarrow D_{\alpha}$ Define $f: C \rightarrow \times D_{\alpha}$ by $f(x)=\left(f_{\alpha}(x)\right)$. This is an isotone function; therefore the image $f(C)$ is directed. It projects upon a cofinal set in each factor $D_{\alpha}$ since $f_{\alpha}$ is convergent. Thus $f(C)$ is a subdirect product of $\left\{D_{\alpha}\right\} . f: C \rightarrow f(C)$ is convergent by $1.2, C \geqq f(C)$.

If $C$ is a direct product, the relations $f(C) \geqq D_{\alpha}$ imply $f(C) \geqq C$; thus $f(C)$ has the same type as $C$ and is also a direct product. Then (by the same argument) $E \geqq f(C)$ for every subdirect product $E$ of $\left\{D_{\alpha}\right\}$, so $f(C)$ is a free sum of all the subdirect products. Conversely, if there exists a free sum $F$ of all subdirect products $E$, then $F$ has all $D_{\alpha}$ as quotients, and by the preceding paragraph, $F$ is a quotient of every directed set that has all $D_{\alpha}$ as quotients. This completes the proof.

As for negative results, it seems instructive to begin with simple modifications of 2.3. They will not take us far, and we omit details. (1) Suppose $P$ is a direct product of $m$ directed sets, and $W(m)$ is not a quotient of $P$. Then $P$ is a quotient of the weak product of a subfamily of $n$ directed sets, $n<m$. (2) If the smallest such $n$ is infinite, $W(n)$ is a quotient of $P$. Hence (3) if all the factors have lower characters $>m, P$ must be a finite partial product. Also (4) in case $m=\aleph_{1}$, if $\omega_{1}$ is not a quotient, then (1) and (2) show that $P$ is a direct product of a countable subfamily. To summarize, if $P$ is expressed, not wastefully, as a direct product of $m \geqq \boldsymbol{\aleph}_{0}$ directed sets, $P$ must have at least one quotient $W(n)$, where $n$ is an infinite cardinal $\leqq m$. Only for $m<\boldsymbol{\aleph}_{2}$ have we shown that $W(m)$ must be a quotient. We now set about showing that having all of $W\left(\boldsymbol{\aleph}_{0}\right), \ldots, W(m)$ as factors is not a sufficient condition for the existence of a direct product.

The bursting number of a directed set $D$ is the smallest cardinal exceeding the power of every bounded subset of $D$. It is obviously not an invariant of cofinal type. Every set $\Delta_{0}(A)$ has bursting number $\aleph_{0}$; for each of its members (being a finite subset of $A$ ) bounds only a finite set. The bursting number of a cardinal set $W(m)$ is $m$.

2.6. If $D$ is a quotient of a product $B \times C$, and the lower character of $B$ is greater than the bursting number of $D$, then $D$ is a quotient of $C$.

Proof. Let $g: D \rightarrow B \times C$ be a Tukey function. Then the second coordinate $g_{2}: D \rightarrow C$ must be a Tukey function, i.e. every unbounded set $S$ in $D$ has unbounded image in $C$. If this were false, $S$ would have unbounded 
image in $B$; this implies that the power of $S$ is greater than the bursting number $m$ of $D$, and $S$ contains an unbounded set $T$ of power $m$. But then $T$ has bounded image in $B$ and also in $C$, hence in $B \times C$, a contradiction.

2.7. If $A$ is an uncountable set, then $\Delta_{0}(A)$ is not a quotient of any strong product of pairwise nonisomorphic cardinal sets.

Proof. Such a strong product either has uncountable lower character or has the form $D \times W\left(\aleph_{0}\right)$, where $D$ has uncountable lower character. As $\Delta_{0}(A)$ is obviously not a quotient of $W\left(\aleph_{0}\right)$, the result follows from 2.6.

The reducing number of a directed set $D$, not of zero type, is the smallest cardinal $m$ such that $D$ is a union of $m$ directed subsets none of which has $D$ as a quotient. $W(m)$ is a quotient of $D$, by 1.5. (In effect, we used this in 2.3.) It is not known whether the reducing number must be regular; it is easy to verify that it is no greater than $\operatorname{ch}(u(D))$. It is not known whether it is an invariant of cofinal type, but it is invariant for complete sets.

2.8. Two complete directed sets of the same nonzero cofinal type have the same reducing number, and no other equivalent set has a smaller reducing number.

Proof. Given equivalent sets $D$ and $E, E$ being complete, there is an isotone convergent function $f: D \rightarrow E$. Let $D$ be a union of $m$ directed sets $D_{\alpha}$ none of which has $D$ as a quotient. The sets $f\left(D_{\alpha}\right)$ are directed, and each is cofinal in the set $E_{\alpha}$ of all predecessors of elements of $f\left(D_{\alpha}\right)$. Thus no $E_{\alpha}$ has $D$ as a quotient; and no $E_{\alpha}$ has $E$ as a quotient. Since $f(D)$ is cofinal in $E, E$ is the union of the sets $E_{\alpha}$. Thus the reducing number of $E$ is no greater than that of $D$.

2.9. If $D$ is a complete quotient of a product $B \times C$, and the upper character of $B$ is less than the reducing number of $D$, then $D$ is a quotient of $C$.

Proof. Let $f: B \times C \rightarrow D$ be an isotone convergent function, and $B_{0}$ a cofinal subset of $B$ of power less than the reducing number of $D$. The sets $\{b\} \times C, b$ in $B_{0}$, are directed; so is the set $D_{b}$ of all predecessors of elements $f(b, c)$ in $D$. The union of the $D_{b}$ is all of $D$, so one of them has $D$ as a quotient; but all of them are quotients of $C$.

2.10. The cardinal quotients of a weak product of cardinal sets $W\left(m_{\alpha}\right)$ $(\alpha \in A)$ are just the $m_{\alpha}$ and the regular cardinals not greater then the power of $A$.

Proof. In view of 2.2 , the weak product $W$ has at least these cardinal quotients. Suppose $n$ is another regular cardinal, greater than the power of $A$ and different from all $m_{\alpha}$. Partition $A$ into the set $A_{0}$ of those $\alpha$ such that $m_{\alpha}<n$ and the remainder $R=A_{1}$. Then $W=W_{0} \times W_{1}$, where $W_{i}$ is a weak product of $\left\{W\left(m_{\alpha}\right): \alpha \in A_{i}\right\}$ formed on the same base points $b_{\alpha}$ as is $W$. Let $h: W_{1} \rightarrow \Delta_{0}(R)$ be the projection $\left(h(x)=\left\{\alpha: x_{\alpha} \neq b_{\alpha}\right\}\right)$. Now the upper character of $W_{0}$ is a sum of fewer than $n$ cardinals less than $n$; 
so by $2.9, W(n)$ is not a quotient of $W$ unless it is a quotient of $W_{1}$. Suppose then that $g: W(n) \rightarrow W_{1}$ is a Tukey function. Its image must be (cf. 1.5) a set of $n$ elements of $W_{1}$ having no bounded $n$-element subset. Any $n$ elements of $X W\left(m_{\alpha}\right)\left(\alpha \in A_{1}\right)$ are coordinatewise bounded; so hg must be a Tukey function. That is, $W(n)$ is a quotient of $\Delta_{0}(R)$, which is absurd.

2.11. $A(\lambda+1)$-product of $n$ cardinal sets $W\left(m_{\alpha}\right)(\alpha \in A)$ has no cardinal quotient $r$ such that $n^{\aleph_{\lambda}}<r<m$ for all $\alpha$.

Proof. Let $D$ be the $(\lambda+1)$-product formed with base points $b_{\alpha}$. Let $h: D \rightarrow \Delta_{\lambda+1}(A)$ be the projection. Suppose that $g: W(r) \rightarrow D$ is a Tukey function. Just as in 2.10 , hg is a Tukey function. But $r$ exceeds the upper character of $\Delta_{\lambda+1}(A)$, a contradiction.

2.12. TheOREM. No uncountable set of pairwise nonisomorphic cardinal sets has a direct product.

Proof. For an uncountable set of cardinal sets $W\left(m_{\alpha}\right)(\alpha \in A)$, the strong product does not have $\Delta_{0}(A)$ as a quotient (2.7). Moreover, if $B$ is any uncountable subset of $A, \Delta_{0}(B)$ is not a quotient of the strong product, hence not a quotient of the supposed direct product.

A subdirect product $C$ of $\left\{D_{\alpha}: \alpha \in B\right\}$ will be said to be decapitated, by $u \in \times D_{\alpha}$, if for each $x$ in $C$ the set $F(x)$ of indices $\alpha$ such that $x_{\alpha}$ is a successor of $u_{\alpha}$ is finite. This $F$ is an isotone convergent function from $C$ to $\Delta_{0}(B)$.

Supposing the cardinal sets $D_{\alpha}=W\left(\boldsymbol{\aleph}_{\alpha}\right) \quad(\alpha \in A)$ have a direct product $P$, we may assume that $P$ is a subdirect product, by 2.5. Partition the index set $A$ into the set $B=A_{0}$ of all indices having only countably many predecessors in $A$ and the possibly empty remainder $A_{1}$. Split the strong product $\times D_{\alpha}$ accordingly as $X_{0} \times X_{1}, X_{i} \times\left\{D_{\alpha} ; \alpha \in A_{i}\right\}$. The projections $P_{i}$ of $P$ in $X_{i}$ are subdirect products, and (in particular) $P_{0}$ is a quotient of $P$.

We may assume $P_{0}$ is complete, by adjoining predecessors in $X_{0}$ if necessary. Let $Y$ be a weak product of $\left\{D_{\alpha}: \alpha \in B\right\}$, with base points $b_{\alpha}$. Then $Y \times X_{1}$ admits convergent mappings to all $D_{\alpha}$, hence to $P$ and to $P_{0}$; there is even an isotone convergent function $h: Y \times X_{1} \rightarrow P_{0}$.

If 0 and 1 occur among the indices $\alpha$, we treat them exceptionally; define $u_{0}$ and $u_{1}$ are arbitrarily chosen elements of $D_{0}, D_{1}$. For every other $\alpha \in B$, consider the set of all $(y, x) \in Y \times X_{1}$ such that $y_{\alpha}=b_{\alpha}$. This is a directed set and a product of three factors $Z_{1} \times Z_{2} \times X_{1}$, where $Z_{1}$ (respectively $Z_{2}$ ) is a weak product of fewer than $\aleph_{\alpha}$ cardinal sets smaller (respectively larger) than $D_{\alpha} . h_{\alpha}$ maps it isotonically into $D_{\alpha}$. The image cannot (2.6 and 2.9) be cofinal unless $D_{\alpha}$ is a quotient of $Z_{2}$. By 2.10, $D_{\alpha}$ is not a quotient of $Z_{2}$; thus some element $u_{a}$ of $D_{\alpha}$ is greater than all these values of $h_{\alpha}$. The function $u$ now decapitates $P_{0}$; for $h\left(Y \times X_{1}\right)$ is cofinal 
in $P_{0}$, and $h(y, x)$ can have $\alpha$ th coordinate as large as $u_{\alpha}$ only for those $\alpha$ for which $y_{\alpha} \neq b_{\alpha}$ and perhaps for $\alpha=0,1$. Then $\Delta_{0}(B)$ is a quotient of $P$, a contradiction.

REMARK. This proof uses only two special subdirect products. It seems odd, offhand, that they are not the strong and the weak product. One may verify that those two can be used at least in case the number of cardinals smaller than the number of factors, $m$, is less than $m$.

3. Point spectrum. The set $Q(D)$ of cardinal quotients of a directed set $D$ will be called the point spectrum of $D$. The point spectrum determines the lower character, which is its smallest element (1.6). To prove that $Q(D)$ determines the upper character, we shall have to assume part of the generalized continuum hypothesis.

If $E$ is a subset of $D$, the directed set of all bounded (in $D$ ) subsets of $E$ is a quotient of $D$ which we may call the restricted quotient $D \mid E$. (For a Tukey function from $D \mid E$ to $D$, take each bounded set to one of its bounds.)

3.1. Theorem. Assume $2^{\boldsymbol{\aleph}_{\alpha}}<\boldsymbol{\aleph}_{\alpha+\omega}$ for all $\alpha$. Then the upper character of any directed set of nonzero type is the least upper bound of its point spectrum.

Proof. Suppose there is a counterexample, and choose a counterexample $D$ whose upper character $m$ is as small as possible. In view of 1.3 and 1.7, $m$ is singular. Let $E$ be a cofinal subset of power $m$, represented as a union of fewer than $m$ sets $E_{\alpha}$ each smaller than $m$. The upper characters of the quotients $D \mid E_{\alpha}$ are not bounded by any cardinal $n<m$, for then each $E_{\alpha}$ would be a union of $n$ bounded sets and $D$ would have a smaller cofinal subset. On the other hand, from the assumption about cardinals, each $D \mid E_{\alpha}$ has upper character less than $m$. By the choice of $m$ as a minimum, the directed sets $D \mid E_{\alpha}$, and therefore also $D$, have cardinal quotients arbitrarily near $m$.

3.2. Corollary. On the hypothesis $2^{\aleph_{\alpha}}<\boldsymbol{\aleph}_{\alpha+\omega}$, every directed set of nonzero noncardinal type has two or more different cardinal quotients.

Proof. The lower and upper character must differ.

3.3. Theorem. If $D$ is a directed set and $m$ a cardinal number which is a limit of cardinal quotients of $D$, then for some cardinal $n$ satisfying $m \leqq n$ $\leqq 2^{m}, \operatorname{ch}(n) \in Q(D)$.

Proof. By 2.5, $D$ has a quotient $E$ which is subdirect product of sets $\omega_{\alpha}$ where $\boldsymbol{\aleph}_{\alpha}$ converges to $m$. The upper character $n$ of $E$ must be at least $m$, and the power of $E$ is at most $m^{m}=2^{m}$. By 1.7, $\operatorname{ch}(n) \in Q(D)$.

This is the first of several theorems which we can state and prove without any continuum hypothesis but cannot well use without some such 
hypothesis. 3.3 can be sharpened in case $m$ is an $\omega$-limit (i.e. $\operatorname{ch}(m)=\boldsymbol{\aleph}_{0}$ ); from 2.4, $Q(D)$ must include either $\aleph_{0}$ or $\operatorname{ch}(u(S))$, where $S$ is the strong product of the sets $\omega_{\alpha}$. We do not know what $\operatorname{ch}(u(S))$ is, nor whether it is determined by $m$. We can choose $S$ as the strong product of a simple sequence of factors $\omega_{\alpha} \rightarrow m$; and then $\operatorname{ch}(u(S))>m$, as follows. There are factorizations $S=I_{\alpha} \times J_{\alpha}$, where $u\left(I_{\alpha}\right)<m$ and $l\left(J_{\alpha}\right)$ is arbitrarily near $m$. Then $u(S)=u\left(J_{\alpha}\right)$, so $\operatorname{ch}(u(S))$ is not less than $m$ (by 1.4); and it is not equal to $m$, since $m$ is singular.

A natural digression: what is the upper character of a strong product in general?

3.4. The upper character of a strong product of directed sets $D_{\alpha}$ is greater than any sum $\sum k_{\alpha}$ of cardinals $k_{\alpha}$ each less than $u\left(D_{\alpha}\right)$.

Proof. Let $S$ be a set of $\sum k_{\alpha}$ elements of $X D_{\alpha}$, expressed as a union of sets $S_{\alpha}$ of $k_{\alpha}$ elements. No $S_{\alpha}$ has cofinal coordinate projections in $D_{\alpha}$; thus some $x_{\alpha}$ is not succeeded by the $\alpha$ th coordinate of any element of $S_{\alpha}$; this gives us an element $x$ of the strong product which has no successor in $S$.

This proposition gives the complete answer if the generalized continuum hypothesis is assumed, for on that hypothesis, König's theorem is sharp; every product of infinite cardinals $m_{\alpha}$ is the smallest number greater than all $\sum k_{\alpha}\left(k_{\alpha}<m_{\alpha}\right)$. This result, one must suppose, is known, though I have not found a reference. At any rate (with the hypothesis), one can write out explicit rules determining all products, and check the present assertion by cases. The referee points out that the converse holds also; the statement that König's theorem is sharp is equivalent to the generalized continuum hypothesis. We have:

3.5. Assuming the generalized continuum hypothesis, the upper character of a strong product is the product of the upper characters of the factors.

The following restatement of 3.3 will be convenient. Call a set $S$ of cardinal numbers low at a cardinal $m$ if $m$ is a limit point of $S$ and $S$ does not include $\operatorname{ch}(n)$ for any $n$ in $\left(m, 2^{m}\right]$. Then (3.3) if $Q(D)$ is low at $m$, $\operatorname{ch}(m) \in Q(D)$.

We call a set $S$ of ordinals heavy at an ordinal $\alpha$ if, with respect to the order topology in $W(\alpha), S \cap W(\alpha)$ cannot be represented as a topological sum of bounded subsets. The results on heaviness call for some preliminary remarks. We have theorems to the effect that $\operatorname{ch}(m) \in Q(D)$ if $(1) Q(D)$ is heavy at $m$, or (2) the set of cardinals at which $Q(D)$ is low is heavy at $m$. However, (2) is marred by an extra hypothesis: $m$ is not (weakly) inaccessible (i.e. not a regular limit cardinal). On the other hand, (1) is simple, flawless, and almost vacuous; we shall show that it cannot apply except to certain highly inaccessible numbers.

3.6. A set $S$ of infinite cardinals is heavy at a cardinal $m$ if and only if $S$ meets every closed unbounded subset of $W(m)$. 
Proof. If $S \cap W(m)$ is contained in the relative complement of an unbounded closed set $K$, it is the sum of its intersections with the (bounded) maximal open intervals disjoint from $K$. Conversely, suppose $S \cap W(m)$ is a sum of bounded sets $S_{\alpha}$. For each $\alpha$, some of the upper bounds of $S_{\alpha}$ are not in $S$, since some of them are noncardinal. Let $b_{\alpha}$ be the smallest such bound. The set $J$ of all $b_{\alpha}$ is then disjoint from $S$, and so is its closure $K$, since $S \cap W(m)$ is the topological sum of the $S_{\alpha}$. If $K$ is unbounded, we are done. If $K$ is bounded, so is $S \cap W(m)$, and we are done (since $W(m)$ has no greatest element).

3.7. If some set of infinite cardinals is heavy at $m$ then $m$ is a limit cardinal and $\operatorname{ch}(m)>\boldsymbol{\aleph}_{0}$.

This is obvious. It follows that $W(m)$ is countably compact; every two unbounded closed sets $K, L$ have unbounded intersection; and thus if $S$ is heavy at $m$, so is $S \cap K$.

A $\rho$-number [4] is a cardinal at which the set of all smaller inaccessible cardinals is heavy. It is known [4] (and not hard to prove) that $\rho$-numbers themselves are inaccessible.

3.8. If some set $S$ of regular alephs is heavy at $m$ then $m$ is a $\rho$-number.

Proof. By 3.7, the limit cardinals in $W(m)$ form a closed unbounded set $K$. $S \cap K$ is a set of inaccessible predecessors of $m$, heavy at $m$.

3.9. Theorem. If $Q(D)$ is heavy at $m$, then $m \in Q(D)$.

Proof. By 2.5, $D$ must have a quotient $P$ that is a subdirect product of $\{W(n): n \in S\}$, where $S$ is a set of cardinals in $W(m)$ that is heavy at $m$. For any $x$ in $P$, we shall show that there is $s \in W(m)$ such that for arbitrarily large $n$ in $S, x_{n} \leqq s$. If this were not true, then for every $t$ in $W(m)$ there would be a least $u(t) \in W(m)$ such that for $n \geqq u(t)$ in $S, x_{n} \geqq t$. This $u$ is a continuous function. Hence the set $T$ of all $t$ such that $u(t) \leqq t$ is closed. $T$ is also unbounded; for if $u(t)>t$, and iteration yields, for all $n, u^{n+1}(t)>u^{n}(t)$, then the values $u^{n}(t)$ converge to a limit $v$ which must belong to $T$. It follows that $S$ and $T$ have a common element $n$, where $u(n) \leqq n$ and $x_{n} \geqq n$, an absurdity.

Define $f(x)$ to be the smallest such $s$. Then $f: P \rightarrow W(m)$ is isotone and convergent. Since $m$ is regular, the theorem is proved.

A more frequently applicable relation between $Q(D)$ and $m$ is that the set of cardinals at which $Q(D)$ is low is heavy at $m$; we say $Q(D)$ drags at $m$.

3.10. Theorem. If $Q(D)$ drags at a singular cardinal $m$, then $\operatorname{ch}(m)$ $\in Q(D)$.

Proof. We have $\operatorname{ch}(m)=l<m$. There is an ascending sequence of cardinals cofinal in $W(m)$ that is order-isomorphic with $W(l)$. Its closure (in $W(m)$ ) is still order-isomorphic with $W(l)$. Since (by 3.7) $l>\boldsymbol{\aleph}_{0}$, the 
nonisolated members of this set still form a closed unbounded subset $L$ of $W(m)$; and each element $n$ of $L$ has $\operatorname{ch}(n)<l$.

Let $J$ be the set of members of $L$ at which $Q(D)$ is low. ( $J$ is heavy at $m$.) For each $j \in J$, take a subset of $Q(D)$ of power less than $l$ having $j$ as a limit. Uniting these sets, we get a subset $I$ of $Q(D)$, order-isomorphic with $W(l)$, which is low at every element of $J$. By $2.5, D$ has a quotient $P$ that is a subdirect product of $\{W(i): i \in I\}$. For each $j$ in $J$, consider the projection $P_{j}$ of $P$ in the strong product $\times\{W(i): i<j, i \in I\}$. The upper character of $P_{j}$ is exactly $j$, for it must exceed all these cardinals $i$, and the other cardinals up through $2^{j}$ are excluded as nonquotients of $D$. Thus $P_{j}$ has a cofinal subset which is a union of $\operatorname{ch}(j)$ sets $H_{\alpha}$, each of power less than $j$. Each $H_{\alpha}$ has power less than $k$ for some $k=k(\alpha)<j$ in $I$. Hence there exists $x=x(j, \alpha)$ in $\times\{W(i): i \in I\}$ such that for each $y$ in $H_{\alpha}, y_{i} \leqq x_{i}$ whenever $k(\alpha) \leqq i<j$ and $i \in I$. Selecting such $x(j, \alpha)$ for all $j$ and $\alpha$, we have $l$ elements of the strong product. Some member $r$ of $I$ is greater than $l$; and for $i \geqq r$ in $I$, all the coordinates $x_{i}(j, \alpha)$ have a common bound $b_{i}$. Define $b_{i}$ in an arbitrary manner for $i<r$ in $I$.

For any $y$ in $P$, for $j>r$ in $J$, there exists $k=k_{y}(j)<j$ in $I$ such that the coordinates of $y$ between the $k$ th coordinate and the gap at $j$ are less than or equal to those of some $x(j, \alpha)$; if we put $k \geqq r$, we may conclude $y_{i} \leqq b_{i}$ when $k \leqq i<j$. Consider the function $k_{y}$ on the set of all $j>r$ in $J$. We shall show that on some cofinal subset of $J, k_{y}$ is bounded away from $m$. Suppose the contrary. Then for ordinal $t<m$, there is a least $u(t)<m$ such that for all $j \geqq u(t)$ in $J, k_{y}(j) \geqq t$. As in the proof of 3.9 , $u$ is continuous, and the set $T$ of all $t>r$ such that $u(t) \leqq t$ is closed and unbounded. Since $J$ is heavy at $m, J$ and $T$ have a common element $j$. Here $u(j) \leqq j$, whence $k_{y}(j) \geqq j$, a contradiction. This shows that $k_{y}$ is bounded on a cofinal set.

If $v$ is an ordinal between $r$ and $m$ such that $k_{y}$ cofinally has values $\leqq v$, then $y_{i} \leqq b_{i}$ for all $i \geqq v$ in $I$. Thus for every $y$ in $P$ there is a least index $f(y) \in I$ such that $y_{i} \leqq b_{i}$ after $f(y)$. We have $f: P \rightarrow I$ isotone and convergent, and $I$ is cofinal in $W(m)$. This completes the proof.

3.11. TheоReм. Assuming the generalized continuum hypothesis, for every set $S$ of at most $\boldsymbol{\aleph}_{1}$ regular alephs which contains $\operatorname{ch}(m)$ whenever $S$ is low at $m$ or drags at $m$, there exists a directed set $D$ such that $Q(D)=S$.

Proof. We consider four cases according as $S$ does or does not include $\boldsymbol{\aleph}_{0}$ and $\boldsymbol{\aleph}_{1}$. If $S$ includes both, let $D$ be a weak product of all $W(m), m \in S$, and if $S$ includes neither, let $D$ be their strong product. The first case is settled by 2.10 . In the second case, clearly $S \subset Q(D)$. For any regular cardinal $m$ not in $S, D$ is the product $D_{1} \times D_{2}$, where $D_{1}$ is the strong product of all $W\left(m_{\alpha}\right), m_{\alpha} \in S, m_{\alpha}<m$, and $D_{2}$ the strong product of the 
remaining factors. $l\left(D_{2}\right)$ exceeds the bursting number $m$ of $W(m)$ (or $D_{2}$ has zero type); so by 2.6 (or trivially) we need only show that $m \notin Q\left(D_{1}\right)$. Now (given the generalized continuum hypothesis) a regular cardinal $m$ always exceeds any product of at most $\boldsymbol{\kappa}_{1}$ pairwise distinct smaller infinite cardinals unless $m=\boldsymbol{\aleph}_{\lambda+1}$ where $\boldsymbol{\aleph}_{\lambda}$ is a limit of the factors, so that $\operatorname{ch}\left(\boldsymbol{\aleph}_{\lambda}\right)$ $\leqq \boldsymbol{\aleph}_{1}$. As $S$ does not include $\boldsymbol{\aleph}_{0}$ or $\boldsymbol{\aleph}_{1}, S$ cannot be low at $\boldsymbol{\aleph}_{\lambda} ; m \in S$.

If $\aleph_{0}$ is not in $S$ and $\aleph_{1}$ is in $S$, let $D$ be a 1-product of all $W\left(m_{\alpha}\right), m_{\alpha} \in S$. Then $Q(D)$ contains $S$, and $\aleph_{0} \notin Q(D)$ since $l(D)=\aleph_{1}$. For any larger regular $m$ not in $S$, factor $D=D_{1} \times D_{2}$ as in the preceding case. Again the cardinal number of $D_{1}$ is less than $m$; so by 2.9 , we need only $m \notin Q\left(D_{2}\right)$, which holds because of 2.11 .

Finally there is the case $\aleph_{0} \in S, \aleph_{1} \notin S$. Then $S$ is a set of power $\aleph_{1}$ or less, not dragging at any cardinal (use 3.7). The union $S^{\prime}$ of $S$ and the set of all cardinals at which $S$ is low is not heavy at any cardinal (use 3.8). By induction on the order type of $S^{\prime}$ one sees that $S^{\prime}$ can be expressed as a topological sum of countable sets $J_{\beta}$. Let $I_{\beta}=J_{\beta} \cap S$. The members of countable set $I_{\beta}$ can be indexed in a one-to-one manner as $m(\beta, n), n$ a positive integer. For each positive integer $r$, let $D_{r}$ be the subset of $X\{W(m): m \in S\}$ consisting of all $x$ such that $x_{m}=0$ for those $m=m(\beta, n)$ such that $n>r$. Note that $D_{r}$ is a strong product of different cardinal sets not including $W\left(\boldsymbol{\aleph}_{1}\right)$. Let $D$ be the union of all $D_{r}$. $D$ is directed; in fact, it is a subdirect product of the sets $W(m), m$ in $S$, so $Q(D)$ contains $S$.

Consider any regular cardinal $m$ not in $S$. Suppose there were were a Tukey function $g: W(m) \rightarrow D$. Since $\boldsymbol{\aleph}_{0} \in S$, there must be $m$ values of $g$ in one $D_{r}$, and $m \in Q\left(D_{r}\right)$. As before, $D_{r}$ is the product of two factors $C_{1}, C_{2}$, which are respectively strong products of certain cardinal sets smaller than $m$ and larger than $m$. By 2.6 we need only show $m \notin Q\left(C_{1}\right)$. $C_{1}$ has power less than $m$ (as before) unless $m=\boldsymbol{\kappa}_{\lambda+1}$, where $\boldsymbol{K}_{\lambda}$ is a limit of the factors. In this case $S$ must be low at $\boldsymbol{\kappa}_{\lambda}$, since $m \notin S$. But every cardinal $\boldsymbol{\aleph}_{l}$ at which $S$ is low is in some $J_{\beta}$. Only finitely many members of $I_{\beta}$ index factors of $C_{1}$; the indices of the other factors of $C_{1}$ do not have $\boldsymbol{K}_{\lambda}$ as a limit point, and we have a contradiction.

4. Standard sets. The symbol $\Delta_{\lambda \mu}$ (of Tukey [7]), where $\lambda$ and $\mu$ are ordinals with $\lambda \leqq \mu$, denotes any directed set $\Delta_{\lambda}(A), A$ having power $\boldsymbol{\aleph}_{\mu}$. We define $\Delta_{\lambda \mu}^{*}$, for all pairs $\lambda \leqq \mu$ except $(\lambda, \lambda)$ when $\aleph_{\lambda}$ is singular, as the directed set of all bounded subsets of $W\left(\boldsymbol{\aleph}_{\mu}\right)$ having power $<\boldsymbol{\aleph}_{\lambda}$. Evidently $\Delta_{\lambda_{\mu}}^{*}$ is "usually" the same as $\Delta_{\lambda}\left(W\left(\boldsymbol{N}_{\mu}\right)\right)$; precisely, this holds if and only if $\operatorname{ch}\left(\boldsymbol{\aleph}_{\mu}\right) \geqq \boldsymbol{\aleph}_{\lambda}$.

As was indicated in the Introduction, we shall establish a characterization of the $\Delta_{\lambda \mu}^{*}$ depending on the generalized continuum hypothesis, and, motivated by that, call them standard sets. Without continuum hypothesis, it may be that the sets $\Delta_{\lambda \mu}^{*}$ are not very useful standards, and 
it may be that the characterization is "usually " vacuous or characterizes some different directed sets. At any rate, we agree that the term "standard set" shall refer to precisely the sets $\Delta_{\lambda \mu}^{*}$. For some of them the results do not require any continuum hypothesis.

The principal bursting number bu(D) of a directed set $D$ is the smallest bursting number of any cofinal subset of $D$.

4.1. The principal bursting number is an invariant of cofinal type.

Proof. Use the fact ([3] or [7]) that equivalent directed sets $D, E$, can be simultaneously embedded as cofinal subsets of a third directed set $F$. If $D$ has a cofinal subset $D_{1}$ no $m$ of whose elements are bounded, then $E$ has a cofinal subset $E_{1}$ any $m$ of whose elements exceed $m$ elements of $D_{1}$.

If $\operatorname{bu}(D)=l(D)$, then $D$ is universal in character, i.e, for a given directed set $E$ to be a quotient of $D$, the necessary conditions $l(E) \geqq l(D), u(E)$ $\geqq u(D)$, are also sufficient. For (assuming, as we may, that bu(D) is the bursting number) there exists a one-to-one function $g$ from a cofinal subset of $E$ to $D$, and $g$ must be a Tukey function, for a subset of its domain that is large enough to be unbounded is so large that its image must be unbounded.

We shall call $D$ a band set if bu $(D)=l(D)$. Band sets coincide with sets universal in character if the generalized continuum hypothesis is assumed; this is essentially in [6], in view of 1.4 above.

4.2 (Schmidt). $l\left(\Delta_{\lambda_{\mu}}\right)=\operatorname{ch}\left(\boldsymbol{\aleph}_{\lambda}\right)$.

4.3. $u\left(\Delta_{\lambda_{\mu}}\right) \geqq \boldsymbol{\aleph}_{\mu}$. For equality, a necessary condition is $\operatorname{ch}\left(\boldsymbol{\aleph}_{\mu}\right) \geqq \boldsymbol{\aleph}_{\lambda}$, and a sufficient condition is $\boldsymbol{\aleph}_{\mu}^{m}=\boldsymbol{\aleph}_{\mu}$ for all $m<\boldsymbol{\aleph}_{\lambda}$. Assuming the generalized continuum hypothesis, these conditions are equivalent.

Proof. Schmidt proved [6] all of 4.2 and 4.3 except the necessity of $\operatorname{ch}\left(\boldsymbol{\aleph}_{\mu}\right)$ $\geqq \boldsymbol{\aleph}_{\lambda}$ without continuum hypothesis. If $\operatorname{ch}\left(\boldsymbol{\aleph}_{\mu}\right)=m<\boldsymbol{\aleph}_{\lambda}$, then a set $S$ of $\aleph_{\mu}$ elements of $\Delta_{\lambda \mu}=\Delta_{\lambda}(A)$ cannot be cofinal. For $S$ is a union of $m$ subsets $S_{\alpha}$, each having power less than $\aleph_{\mu}$ and having union of power less than $\boldsymbol{\aleph}_{\mu}$. Thus no $S_{\alpha}$ covers $A$; and one can choose a set of $m$ elements of $A$ which is not contained in the union of any $S_{\alpha}$, and therefore has no successor in $S$.

4.4. $\mathrm{bu}\left(\Delta_{\lambda_{\mu}}\right) \geqq \boldsymbol{\aleph}_{\lambda}$, with equality at least in case $u\left(\Delta_{\lambda_{\mu}}\right)=\boldsymbol{\aleph}_{\mu}$.

Proof. Let $A$ have power $\boldsymbol{\aleph}_{\mu}$. If $\boldsymbol{\aleph}_{\lambda}$ is regular, every subset of $\Delta_{\lambda}(A)$ of smaller power is bounded. Suppose $\boldsymbol{K}_{\lambda}$ is singular. Then for every regular cardinal $m$ between $\operatorname{ch}\left(\boldsymbol{\kappa}_{\lambda}\right)$ and $\boldsymbol{\aleph}_{\lambda}$, and $m$ elements of $\Delta_{\lambda}(A)$ include $m$ elements of power bounded below $\boldsymbol{K}_{\lambda}$, which form a bounded set. This proves the inequality. Now suppose there is a cofinal set $D=\left\{d_{\alpha}\right\}$ of $\boldsymbol{\aleph}_{\mu}$ elements of $\Delta_{\lambda}(A)$, indexed by ordinals $\alpha<\omega_{\mu}$. We construct another cofinal set $E=\left\{e_{\alpha}\right\}$ by transfinite recursion. Check first that for each $\alpha$, the preceding sets $e_{\beta}$ cannot cover $A$. This is obvious if $\lambda<\mu$. If $\lambda=\mu$, then since $u\left(\Delta_{\lambda \mu}\right)=\boldsymbol{\aleph}_{\mu}, \boldsymbol{\aleph}_{\mu}$ is regular by 4.3 ; that makes this case obvious 
also. Thus for each $\alpha$, there is $a_{\alpha} \in A$ not belonging to any of the preceding $e_{\beta}$. Let $e_{\alpha}=d_{\alpha} \cup\left\{a_{\alpha}\right\}$. Now $E$ is cofinal, and any $\boldsymbol{\aleph}_{\lambda}$ of its elements have a union of power $\boldsymbol{K}_{\lambda}$; so any $\boldsymbol{\aleph}_{\lambda}$ of its elements are unbounded.

Thus we have Schmidt's theorem in case $\boldsymbol{\aleph}_{\lambda}$ and $\boldsymbol{\aleph}_{\mu}$ are the characters: $\Delta_{\lambda \mu}$ is a band set and is universal in character [6]. In this case, by 4.3, $\Delta_{\lambda \mu}^{*}$ is a set $\Delta_{\lambda_{\mu}}$. Schmidt noted that no continuum hypothesis is needed to prove the theorem in case $\lambda=0$ or $\lambda=\mu$ where $\boldsymbol{K}_{\mu}$ is regular. Without continuum hypothesis, one can add the following result.

4.5. If $\boldsymbol{\aleph}_{\lambda}$ is regular and $\lambda<\mu<\lambda+\omega_{0}$, then $u\left(\Delta_{\lambda_{\mu}}\right)=\boldsymbol{N}_{\mu}$; hence bu $\left(\Delta_{\lambda_{\mu}}\right)$ $=\boldsymbol{\aleph}_{\lambda}$, and $\Delta_{\lambda_{\mu}}$ is universal in character.

Proof. We must exhibit a cofinal subset of $\Delta_{\lambda}(A)$ having the same power $\boldsymbol{\aleph}_{\lambda+k+1}$ as $A$, knowing that this is possible for $\boldsymbol{K}_{\lambda+k}$. (The induction began with $k=0$, and regularity of $\boldsymbol{\aleph}_{\lambda}$ was needed there.) Identify $A$ with $W\left(\omega_{\lambda+k+1}\right)$. For $\omega_{\lambda+k}<\alpha \in A$, there is a set $S_{\alpha}$ of $\boldsymbol{K}_{\lambda+k}$ elements of $\Delta_{\lambda}(W(\alpha))$ which is cofinal in it. $\Delta_{\lambda}(A)$ is just the union of the sets $\Delta_{\lambda}(W(\alpha))$; so the union of the $S_{\alpha}$ is cofinal in it.

We turn to the sets $\Delta_{\lambda_{\mu}}^{*}$. Thus we are considering the same sets $\Delta_{\lambda_{\mu}}$ in the more extensive case $\operatorname{ch}\left(\boldsymbol{\aleph}_{\mu}\right) \geqq \boldsymbol{\aleph}_{\lambda}$, and some different sets when $\operatorname{ch}\left(\boldsymbol{\aleph}_{\mu}\right)$ $<\boldsymbol{\aleph}_{\lambda}$; but we still exclude the case of singular $\boldsymbol{\aleph}_{\lambda}=\boldsymbol{\aleph}_{\mu}$.

4.2* $l\left(\Delta_{\lambda_{\mu}}^{*}\right)=\min \left(\operatorname{ch}\left(\boldsymbol{\kappa}_{\lambda}\right), \operatorname{ch}\left(\boldsymbol{\aleph}_{\mu}\right)\right)$.

This is obvious.

4.3*. $u\left(\Delta_{\lambda_{\mu}}^{*}\right) \geqq \boldsymbol{\aleph}_{\mu}$. For equality, the generalized continuum hypothesis is sufficient.

Proof. Fewer than $\boldsymbol{\aleph}_{\mu}$ sets of powers $\left\langle\boldsymbol{\aleph}_{\lambda} \leqq \boldsymbol{\aleph}_{\mu}\right.$ cannot cover $\boldsymbol{W}\left(\boldsymbol{\aleph}_{\mu}\right)$, unless $\boldsymbol{\aleph}_{\mu}$ is singular and $\boldsymbol{\aleph}_{\lambda}=\boldsymbol{\kappa}_{\mu}$, an excluded case. On the other hand, $\Delta_{\lambda \mu}^{*}$ is the union of $\boldsymbol{\aleph}_{\mu}$ sets $\Delta_{\lambda}(W(\alpha)), \alpha<\omega_{\mu}$, and with the present hypothesis, they have power $\leqq \boldsymbol{N}_{\mu}$,

4.4. $4^{*}$ bu $\left(\Delta_{\lambda_{\mu}}^{*}\right) \geqq \boldsymbol{\aleph}_{\lambda}$. For equality, $u\left(\Delta_{\lambda_{\mu}}^{*}\right)=\boldsymbol{\aleph}_{\mu}$ is sufficient.

Proof. We have the result if $\operatorname{ch}\left(\boldsymbol{\aleph}_{\mu}\right) \geqq \boldsymbol{N}_{\lambda}$. Suppose $\operatorname{ch}\left(\boldsymbol{\aleph}_{\mu}\right)<\boldsymbol{\kappa}_{\lambda}$. For each regular $m>\operatorname{ch}\left(\boldsymbol{\aleph}_{\mu}\right)$, any $m$ elements of $\Delta_{\lambda \mu}^{*}$ include a set $S$ of $m$ elements of some $\Delta_{\lambda}(W(\alpha)), \omega_{\lambda}<\alpha<\omega_{\mu}$. If $\boldsymbol{\aleph}_{\lambda}$ is regular, put $m=\boldsymbol{\aleph}_{\lambda} ; S$ has bounded subsets of power $n$ for every $n<\boldsymbol{K}_{\lambda}$. If $\boldsymbol{\aleph}_{\lambda}$ is singlar, consider $\operatorname{ch}\left(\boldsymbol{\aleph}_{\lambda}\right)<m<\boldsymbol{\aleph}_{\lambda} . S$ has $m$ elements whose powers are bounded away from $\boldsymbol{\aleph}_{\lambda}$, so that they form a bounded set. This proves the inequality. The same construction as in 4.4 proves the equation.

4.5*. If $\boldsymbol{\aleph}_{\lambda}$ is regular then $u\left(\Delta_{\lambda \lambda+\omega_{0}}^{*}\right)=\boldsymbol{\aleph}_{\lambda+\omega_{0}}$.

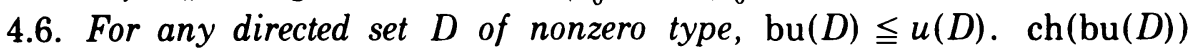
is a cardinal quotient of $D$. Also, every directed set $E$ such that $l(E) \geqq b u(D)$ and $u(E) \leqq u(D)$ is a quotient of $D$.

Proof. $D$ has (by 1.7,1.5) $\operatorname{ch}(u(D))=m$ elements $d_{\alpha}$, every $m$ of which is unbounded. $D$ also has a cofinal set which is a union of $m$ sets $C_{\alpha}=\left\{c_{\alpha \beta}\right\}$ each of power $<u(D)$. For each $\alpha$ and $\beta$, let $e_{\alpha \beta}$ be a common successor 
of $c_{\alpha \beta}$ and $d_{\alpha}$. The $e_{\alpha \beta}$ form a cofinal set; and a subset cannot have power $u(D)$ unless $m$ different first indices $\alpha$ occur. Thus bu $(D) \leqq u(D)$. Next, $D$ has $\operatorname{ch}(\operatorname{bu}(D))$ bounded subsets $B_{\alpha}$ such that no union of $\operatorname{ch}(\mathrm{bu}(D))$ of them is bounded. (Take sets of different power if $\mathrm{bu}(D)$ is a limit cardinal, sets of power 1 otherwise.) Then bounds for the sets $B_{\alpha}$ constitute the set of values of a Tukey function from $W(\operatorname{ch}(b u(D)))$. For the final assertion, any one-to-one function is a Tukey function.

4.7. Every standard set $\Delta_{\lambda \mu}^{*}$ admits two functions $p: \Delta_{\lambda \mu}^{*} \rightarrow W\left(\operatorname{ch}\left(\boldsymbol{\aleph}_{\lambda}\right)\right)$, $q: \Delta_{\lambda_{\mu}}^{*} \rightarrow W\left(\operatorname{ch}\left(\boldsymbol{\aleph}_{\mu}\right)\right)$, such that every subset of power $<\boldsymbol{\aleph}_{\lambda}$ on which $p$ and $q$ are bounded is bounded. If $u\left(\Delta_{\lambda_{\mu}}^{*}\right)=\boldsymbol{\aleph}_{\mu}$, then $\Delta_{\lambda_{\mu}}^{*}$ has a cofinal subset $E_{\lambda_{\mu}}$ whose bounded subsets are precisely the sets of power $\left\langle\boldsymbol{\aleph}_{\lambda}\right.$ on which $p$ and $q$ are bounded.

Proof. If $\boldsymbol{K}_{\lambda}$ is regular, let $p$ be identically 0 . Otherwise identify $W\left(\operatorname{ch}\left(\boldsymbol{K}_{\lambda}\right)\right)$ with a cofinal set of cardinals in $W\left(\boldsymbol{\aleph}_{\lambda}\right)$, and let $p(d)$ be the first of these cardinals exceeding the power of $d$. If $m=\operatorname{ch}\left(\boldsymbol{\aleph}_{\mu}\right) \geqq \boldsymbol{\aleph}_{\lambda}$, let $q$ be identically 0 . Otherwise identify $W(m)$ with a cofinal set of ordinals in $W\left(\boldsymbol{\aleph}_{\mu}\right)$, and let $q(d)$ be the first of these ordinals which is an upper bound for $d$. Then any fewer than $\aleph_{\lambda}$ elements of $\Delta_{\lambda \mu}^{*}$, with their powers bounded away from $\aleph_{\lambda}$ and their least upper bounds bounded away from $\omega_{\mu}$, form a bounded set. If $u\left(\Delta_{\lambda \mu}^{*}\right)=\boldsymbol{\aleph}_{\mu}$, then by $4.4^{*}$ there is a cofinal subset $E_{\lambda \mu}$ every $\boldsymbol{\aleph}_{\lambda}$ of whose elements are unbounded; and clearly a set on which $p$ or $q$ is unbounded is unbounded.

4.8. THEOREM. If $\Delta_{\lambda \mu}^{*}$ has upper character $\boldsymbol{\aleph}_{\mu}$ then it is a free sum of the class of all directed sets $D$ such that $u(D)=\boldsymbol{\aleph}_{\mu}, \operatorname{bu}(D)=\boldsymbol{\aleph}_{\lambda}$, and it is a member of the class.

Proof. As we already know bu $\left(\Delta_{\lambda \mu}^{*}\right)$, we need only show that all these sets $D$ have $\Delta_{\lambda \mu}^{*}$ as a quotient. We may assume that $D$ has bursting number $\boldsymbol{\aleph}_{\lambda}$. By 1.7 and 4.6, there are Tukey functions $i: W\left(\operatorname{ch}\left(\boldsymbol{\aleph}_{\lambda}\right)\right) \rightarrow D$ and $j$ : $W\left(\operatorname{ch}\left(\boldsymbol{K}_{\mu}\right)\right) \rightarrow D$. Let $p$ and $q$ be functions given by 4.7 , and let $F$ be a cofinal subset of $\Delta_{\lambda \mu}^{*}$ of power $\aleph_{\mu}$. Let $h: F \rightarrow D$ be a one-to-one function. For $f \in F$, let $g(f)$ be a common successor of $h(f), i p(f), j q(f)$. On every unbounded subset of $F$, by $4.7, h$ or $i p$ or $j q$ is unbounded. Thus $g$ is a Tukey function.

We remark that the crude statement of 4.8 in the Introduction is correct as hedged; the hypotheses that $D$ has power $\boldsymbol{\kappa}_{\mu}$ and bursting number $\boldsymbol{\kappa}_{\lambda}$ suffice. ( $\Delta_{\lambda \mu}^{*}$ may lack these properties, but it is equivalent in this context (H) to sets that have them.) To see that they suffice, note that the proof of 4.8 applies if $W\left(\boldsymbol{\aleph}_{\lambda}\right)$ and $W\left(\boldsymbol{\aleph}_{\mu}\right)$ are quotients of $D . W\left(\boldsymbol{\aleph}_{\lambda}\right)$ is a quotient, by the proof of 4.6. This disposes of the case $\lambda=\mu$. The remaining case $\lambda<\mu$ requires that the power of $D$ is just its upper character $u$; for if $v$ is a regular cardinal $>u$, any $v$ elements of $D$ (a union of $u$ bounded sets) must contain a bounded set of power $v$. 
4.9. Corollary. Suppose $u\left(\Delta_{\lambda \mu}\right)=u\left(\Delta_{\lambda \mu+1}\right)=\boldsymbol{\aleph}_{\mu+1}$, and $\boldsymbol{\aleph}_{\lambda}>2^{m}$ for all $m<\boldsymbol{K}_{\lambda}$. Then $\Delta_{\lambda \mu}$ is equivalent to $\Delta_{\lambda \mu+1}$.

Proof. In any case $\Delta_{\lambda \mu+1} \geqq \Delta_{\lambda \mu}$; for embedding an $\boldsymbol{\aleph}_{\mu}$-element subset $B$ in an $\boldsymbol{\aleph}_{\mu+1}$-element set $A$ induces a Tukey function from $\Delta_{\lambda}(B)$ to $\Delta_{\lambda}(A)$. 4.8 applies to $\Delta_{\lambda_{\mu}+1}=\Delta_{\lambda_{\mu}+1}^{*}$; and the hypothesis on $\boldsymbol{K}_{\lambda}$ implies that no set $\Delta_{\lambda}(B)$ has an $\boldsymbol{\aleph}_{\lambda}$-element bounded subset. Hence the equivalence.

It seems worth noting that whenever $u\left(\Delta_{\lambda_{\mu}}\right)$ exceeds $\boldsymbol{\aleph}_{\mu}$, it is the same as $u\left(\Delta_{\lambda \mu+1}\right)$, by the method of 4.5 . For 4.9 , though, the value must be just $\boldsymbol{\aleph}_{\mu+1}$. Of course the generalized continuum hypothesis implies that. On that hypothesis, we have determined all $\Delta_{\lambda \mu}$ except those with regular $\boldsymbol{\aleph}_{\lambda}$ $>\operatorname{ch}\left(\boldsymbol{\aleph}_{\mu}\right)$; those have upper character $\boldsymbol{\aleph}_{\mu+1}$ and principal bursting number either $\boldsymbol{K}_{\lambda}$ or $\boldsymbol{\aleph}_{\lambda+1}$.

We can conclude (on the same hypothesis) that band sets and sets universal in character coincide and exist for all possible characters $l, u$ $(l=\operatorname{ch}(l) \leqq \operatorname{ch}(u))$. On the weaker hypothesis of $3.2\left(2^{\kappa_{\alpha}}<\aleph_{a+\omega}\right)$, one can add to 4.8 as follows: for singular $\aleph_{\mu}$, the free sum of all directed sets $D$ such that $u(D)=\operatorname{bu}(D)=\boldsymbol{\aleph}_{\mu}$ is $W\left(\boldsymbol{\aleph}_{\mu}\right)$. I am indebted to the referee for correcting my original misstatement. The proof is (nevertheless) not hard; one need only show that for any regular cardinal $m \neq \operatorname{ch}\left(\boldsymbol{\aleph}_{\mu}\right)$ there is $D$ satisfying $u(D)=\mathrm{bu}(D)=\boldsymbol{\aleph}_{\mu}$ and omitting $m$ from its spectrum. Without any restriction on cardinals, one has

4.10. Any band set $D$ with $l(D)=\aleph_{\lambda}$ and $u(D)=\aleph_{\mu}$ must be equivalent to $\Delta_{\lambda \mu}$.

Proof. $D$ has a cofinal subset $E$ of power $\boldsymbol{\aleph}_{\mu}$ in which the bounded sets are precisely the sets of power $<\boldsymbol{\aleph}_{\lambda}$. For a Tukey function from $E$ to $\Delta_{\lambda}(E)$, take each element $e$ to the singleton $\{e\}$. For a Tukey function in the opposite direction, take each $S \in \Delta_{\lambda}(E)$ to a bound for $S$ in $E$.

As we remarked earlier, the proof of 4.8 proved more; in particular,

4.11. If $u\left(\Delta_{\lambda \mu}^{*}\right)=\boldsymbol{\aleph}_{\mu}$ then $\Delta_{\lambda \mu}^{*}$ is a quotient of every $D$ such that $u(D) \geqq \boldsymbol{\aleph}_{\mu}$, $\operatorname{bu}(D) \leqq \boldsymbol{\aleph}_{\lambda}$, and $\operatorname{ch}\left(\boldsymbol{\aleph}_{\lambda}\right)$ and $\operatorname{ch}\left(\boldsymbol{\aleph}_{\mu}\right)$ are in $Q(D)$.

4.12. $Q\left(\Delta_{\lambda \mu}^{*}\right)$ contains $\operatorname{ch}\left(\boldsymbol{\aleph}_{\lambda}\right), \operatorname{ch}\left(\boldsymbol{\aleph}_{\mu}\right)$, and the regular cardinals between $\boldsymbol{\aleph}_{\lambda}$ and $\boldsymbol{\aleph}_{\mu}$. If it has any other elements they are greater than $\boldsymbol{\aleph}_{\mu}$.

Proof. For every cardinal $m$ between $\aleph_{\lambda}$ and $\aleph_{\mu}, \Delta_{\lambda \mu}^{*}$ contains $m$ distinct singletons, any $m$ of which are unbounded. If $m$ is regular, this yields a Tukey function. For ch $\left(\boldsymbol{\aleph}_{\mu}\right)$, a set of singletons whose union is cofinal in $W\left(\boldsymbol{\aleph}_{\mu}\right)$ gives a Tukey function. If $\boldsymbol{\aleph}_{\lambda}$ is singular, there are $\operatorname{ch}\left(\boldsymbol{\aleph}_{\lambda}\right)$ initial segments of $W\left(\boldsymbol{\aleph}_{\mu}\right)$ whose powers approach $\boldsymbol{\kappa}_{\lambda}$; hence a Tukey function. For any other regular cardinal $m<\boldsymbol{\aleph}_{\lambda}$, let $A$ be a set of $m$ elements of $\Delta_{\lambda \mu}^{*}$. The function $p$ of 4.7 is bounded on an $m$-element subset $B$; the function $q$ is bounded on an $m$-element subset $C$ of $B$. Then $C$ is bounded, and $W(m)$ is not a quotient.

4.13. For each cardinal $\boldsymbol{\aleph}_{\alpha}<\boldsymbol{\aleph}_{\lambda}$, any $\boldsymbol{\aleph}_{\alpha+1}$ elements of $\Delta_{\lambda \mu}^{*}$ include $\boldsymbol{\aleph}_{\alpha+1}$ 
elements, every $\boldsymbol{\aleph}_{\alpha}$ of which is bounded.

Proof. There are $\boldsymbol{\aleph}_{\alpha+1}$ elements all of which are bounded unless at least one of $\operatorname{ch}\left(\boldsymbol{\aleph}_{\lambda}\right), \operatorname{ch}\left(\boldsymbol{\aleph}_{\mu}\right)$ is $\boldsymbol{\aleph}_{\alpha+1}$ (by 4.12). If both are then $\boldsymbol{\aleph}_{\alpha}<l\left(\Delta_{\lambda \mu}^{*}\right)\left(4.2^{*}\right)$ and every $\boldsymbol{\aleph}_{a}$ element is bounded. If $\operatorname{ch}\left(\boldsymbol{\aleph}_{\lambda}\right)$ is $\boldsymbol{\aleph}_{\alpha+1}$ and $\operatorname{ch}\left(\boldsymbol{\aleph}_{\mu}\right)$ is not, then any $\boldsymbol{K}_{\alpha+1}$ elements of $\Delta_{\lambda \mu}^{*}$ have a subset $A$ of $\boldsymbol{\aleph}_{\alpha+1}$ elements on which the function $q$ of 4.7 is bounded. Since $\boldsymbol{\aleph}_{\alpha}<\operatorname{ch}\left(\boldsymbol{\aleph}_{\lambda}\right) \leqq \boldsymbol{K}_{\lambda}$, any $\boldsymbol{K}_{\alpha}$ elements of $A$ are bounded. The remaining case is similar.

Those quotients $\Delta_{\alpha \alpha}^{*}\left(\in K\left(\boldsymbol{\aleph}_{\alpha}\right)\right)$ of $\Delta_{\lambda \mu}^{*}$ for which $\alpha$ is not in the interval $[\lambda, \mu]$ will be called the exceptional cardinal quotients of $\Delta_{\lambda \mu}^{*}$. By 4.12 , there are at most two of them, assuming the generalized continuum hypothesis.

4.14. Theorem. Assuming the generalized continuum hypothesis, the standard quotients $\Delta_{\alpha \beta}^{*}$ of a standard set $\Delta_{\lambda \mu}^{*}$, other than the exceptional cardinal quotients, are just those for which $\lambda<\alpha \leqq \beta \leqq \mu$ and $Q\left(\Delta_{\lambda_{\mu}}^{*}\right)$ includes $\operatorname{ch}\left(\boldsymbol{\aleph}_{\alpha}\right)$ and $\operatorname{ch}\left(\boldsymbol{\aleph}_{\beta}\right)$.

Proof. By 4.11 (with $4.3^{*}$ and $4.4^{*}$ ), all these $\Delta_{\alpha \beta}^{*}$ are quotients. For any other quotients $\Delta_{\alpha \beta}^{*}, \beta>\alpha$ by 4.12 , and necessarily $\beta \leqq \mu$ and $\operatorname{ch}\left(\boldsymbol{\aleph}_{\alpha}\right)$ and $\operatorname{ch}\left(\boldsymbol{\aleph}_{\beta}\right)$ are in $Q\left(\Delta_{\lambda_{\mu}}^{*}\right)$. It remains to rule out the possibility $\alpha<\lambda . \Delta_{\alpha \beta}^{*}$ has a cofinal subset $E$ consisting of at least $\boldsymbol{\aleph}_{\alpha+1}$ elements, every $\boldsymbol{\aleph}_{\alpha}$ of which is unbounded. By $4.13, \Delta_{\lambda \mu}^{*}$ has no such subset. Then a Tukey function from $E$ to $\Delta_{\lambda_{\mu}}^{*}$ would have to take a constant value on a set of power $\aleph_{\alpha+1}$, which is absurd.

Our concluding observation on standard sets is that some are direct products of others.

4.15. If $\operatorname{ch}\left(\boldsymbol{\aleph}_{\mu}\right)=m \leqq \operatorname{ch}\left(\boldsymbol{\aleph}_{\lambda}\right)$, but $\lambda<\mu$, then $\Delta_{\lambda \mu}^{*}$ is a direct product of $W(m)$ and all $\Delta_{\lambda \alpha+1}^{*}, \lambda<\alpha<\mu$.

Proof. These sets are indeed quotients, $W(m)$ by $4.12, \Delta_{\lambda \alpha+1}^{*}\left(=\Delta_{\lambda \alpha+1}\right)$ by the method of 4.9. Suppose $D$ has these quotients. Since they are complete, there are isotone convergent functions $f_{0}: D \rightarrow W(m)$ and $f_{\alpha}: D \rightarrow \Delta_{\lambda \alpha+1}^{*}$. Replace $W(m)$ by a sequence of $\boldsymbol{\aleph}_{\alpha}$ 's order-isomorphic with $W(m)$ converging to $\aleph_{\mu}$; call it $A$. Define $f: D \rightarrow \Delta_{\lambda \mu}^{*}$ as follows: $f(d)$ is the union of all the sets $f_{\alpha}(d)$, for $\alpha<f_{0}(d)$ in $A$. Since $m \leqq \operatorname{ch}\left(\aleph_{\lambda}\right), f(d)$ is indeed an element of $\Delta_{\lambda \mu}^{*}$. $f$ is isotone; and since $\Delta_{\lambda \mu}^{*}$ is the union of the $\Delta_{\lambda \alpha+1}^{*}, f$ is convergent.

5. Additional results and problems. An alternative notation for $\Delta_{\lambda}(A)$ or $\Delta_{\lambda \mu}$ is $\Delta_{\lambda}(m), \lambda$ ordinal and $m$ cardinal, meaning any set $\Delta_{\lambda}(A)$ where $A$ has power $m$.

5.1. If $n$ is an infinite cardinal and $\operatorname{ch}(n)>\boldsymbol{\aleph}_{0}$, then $\Delta_{0}\left(2^{n}\right)$ is equivalent to a strong product whose factors $F(m, \alpha)$ are $n$ copies of $\Delta_{0}\left(2^{m}\right)$ for each $m<n$.

Proof. First, the power of $\Delta_{0}\left(2^{m}\right)$ is $2^{m}$, and the strong product has power $2^{s}$ where $s=\sum n m(m<n)$, which is $n$. As $\Delta_{0}\left(2^{n}\right)$ is universal in character with characters $\boldsymbol{K}_{0}$ and $2^{n}$, we need only show that the strong product, 
or some quotient of it, is the range of a Tukey function from $\Delta_{0}\left(2^{n}\right)$. To this end, identify $\Delta_{0}\left(2^{n}\right)$ with the set of all subsets of the set $W(n)$. For each $\alpha \in W(n)$, let $F(m, \alpha)$ be a copy of $\Delta_{0}\left(2^{m}\right)$, where $m$ is the cardinal number of $\alpha$; and identify $F(m, \alpha)$ with the set of sets of ordinals less than $\alpha$. For each $m<n$, we have no more than $n$ factors $F(m, \alpha)$. Define the coordinate function $g_{\alpha}: \Delta_{0}\left(2^{n}\right) \rightarrow F(m, \alpha)$ to take each $H \subset W(n)$ to the set of all elements of $H$ less than $\alpha$. Then the $g_{\alpha}$ are the coordinates of a Tukey function. For if $\left\{H_{i}\right\}$ is a countably infinite set of subsets of $\mathrm{W}$, any two $H_{i}, H_{j}$ are distinguished by some $g_{\alpha}$; this countable set of $\alpha$ 's has a bound $\beta \in W(n)$, and $g_{\beta}\left(\left\{H_{i}\right\}\right)$ is infinite, hence unbounded.

Let $\pi(n)$ denote a strong product of $n$ copies of $\omega_{0}$ (for infinite $n$ ).

5.2. Theorem. The strong product $\pi(n)$ has $\Delta_{0}(n)$ as a quotient, and it is equivalent to $\Delta_{0}\left(2^{n}\right)$ if $n \geqq 2^{m}$ for all $m<n$ and $\operatorname{ch}(n)>\boldsymbol{\aleph}_{0} . \Delta_{0}\left(\boldsymbol{\aleph}_{1}\right)$ is not a quotient of $\pi\left(\boldsymbol{\aleph}_{0}\right)$.

Proof. The relation $\pi\left(\boldsymbol{\aleph}_{0}\right) \geqq \Delta_{00}$ is trivial. For $n>\boldsymbol{\aleph}_{0}$, identify $\Delta_{0}(n)$ with the set of isolated ordinals in $W(n)$; identify the coordinate indices $\alpha$ with the ordinals $\leqq n$ having character $\boldsymbol{\aleph}_{0}$. For each $\alpha$, select a sequence of ordinals $\beta_{i}(\alpha)$ increasing to $\alpha$. Let $g_{\alpha}(x)$ be 0 for $x<\beta_{1}(\alpha)$ and for $x>\alpha$, but $k$ for $\beta_{k}(\alpha) \leqq x<\beta_{k+1}(\alpha)$. Any infinite set of $x$ 's contains a sequence converging to a limit $\alpha$, and $g_{\alpha}$ is unbounded on such a sequence.

If $n \geqq 2^{m}$ for all $m<n$ then each $\Delta_{0}\left(2^{m}\right)$ is a quotient of $\pi(n)$. Since the strong product of $n$ factors $\pi(n)$ is simply $\pi\left(n^{2}\right)=\pi(n)$, the strong product in 5.1 is a quotient of $\pi(n)$. Thus if the character of $n$ is not $\mathcal{\aleph}_{0}, \pi(n)$ is equivalent to the universal set $\Delta_{0}\left(2^{n}\right)$.

Finally, a convergent function from $\pi\left(\boldsymbol{\aleph}_{0}\right)$ to $\Delta_{0}\left(\boldsymbol{\aleph}_{1}\right)$ would yield a Tukey function from $\Delta_{0}\left(\boldsymbol{\aleph}_{1}\right)$ to $\pi\left(\boldsymbol{\aleph}_{0}\right)$. Its coordinates would be $\boldsymbol{\aleph}_{0}$ integer-valued functions on an uncountable set $X$, not all bounded on any infinite subset of $X$. But these are the coordinates of a function from $X$ into a separable metric space. In such a space, every uncountable set contains a convergent sequence, on which the coordinate functions must be bounded. The proof is complete.

5.3. Corollary. Assuming the generalized continuum hypothesis, $\Delta_{0}\left(\boldsymbol{\aleph}_{\alpha+1}\right)$ is equivalent to $\pi\left(\boldsymbol{\aleph}_{\alpha}\right)$ when $\operatorname{ch}\left(\boldsymbol{\aleph}_{\alpha}\right)>\boldsymbol{\aleph}_{0}$. If $\lambda$ is a limit ordinal or $\lambda=1$, then $\Delta_{0}\left(\boldsymbol{\aleph}_{\lambda}\right)$ is not equivalent to a strong product of sets of smaller upper character.

Proof. The first assertion, and the second for $\lambda=1$, are immediate from 5.2. As for the limit cardinals $\boldsymbol{\aleph}_{\lambda}$, a strong product of sets not having upper character $\aleph_{\lambda}$ cannot have upper character $\boldsymbol{K}_{\lambda}$; for there would have to be either $\boldsymbol{\aleph}_{\lambda}$ factors (not of zero type) or factors with upper characters increasing to $\boldsymbol{\aleph}_{\lambda}$, and in either case the product is too big.

5.4. THEOREM. Every standard set $\Delta_{0}\left(2^{m}\right)$ is equivalent to an ultrafilter of subsets of $W(m)$. 
Proof. The point is that a set $W$ of $m$ elements, for $m$ infinite, has $2^{m}$ independent subsets $S_{a}$; that is, the free Boolean algebra on $2^{m}$ generators can be isomorphically embedded in the algebra of all subsets of $W$. This is the Stone dual of the statement that a Cartesian product of $2^{m}$ two-point spaces is a continuous image of the Stone-Čech compactification of a discrete space of $m$ points, and follows at once from the proof by E. Marczewski (Szpilrajn) [5] that such a product has a dense set of $m$ points.

In $W$, let $\mathcal{S}$ be the collection of all subsets of the form of a union of infinitely many $S_{\alpha}$ minus a union of finitely many $S_{\alpha}$. By independence, the collection $\mathscr{S}$ has the finite intersection property; it generates a filter which is then contained in an ultrafilter $\mathscr{U}$. As $\mathscr{U}$ has power $2^{m}$, it is a quotient of $\Delta_{0}\left(2^{m}\right)$. On the other hand, for a Tukey function from $\Delta_{0}\left(2^{m}\right)$ to $\mathscr{U}$, take any one-to-one function whose values are complements of sets $S_{\alpha}$. Every infinite union of $S_{\alpha}$ 's is in $\mathscr{U}$, so their complements are unbounded.

Many other directed sets of upper character greater than $m$ can be represented by filters in $W(m)$. Here is a sample representation, using instead of Marczewski's independent sets Erdös' almost disjoint sets: more than $m$ subsets of $W(m)$, each having $m$ elements but no two having $m$ common elements [1]. It is convenient to turn the filter upside down.

5.5. If $u(D)=\boldsymbol{\aleph}_{\mu+1}$ and $\operatorname{bu}(D) \leqq \operatorname{ch}\left(\boldsymbol{\aleph}_{\mu}\right)$, then $D$ is equivalent to an ideal of subsets of $W\left(\boldsymbol{\aleph}_{\mu}\right)$.

Proof. $D$ is equivalent to one of its cofinal subsets $E$ of power $\boldsymbol{\kappa}_{\mu+1}$ and bursting number at most $\operatorname{ch}\left(\boldsymbol{\aleph}_{\mu}\right)$. Let $F$ be a family of $\boldsymbol{\aleph}_{\mu+1}$ almost disjoint sets in $W\left(\boldsymbol{\aleph}_{\mu}\right)$. Let $i$ be a one-to-one correspondence between $E$ and

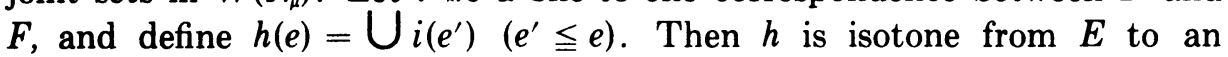
upward-directed family of subsets of $W\left(\boldsymbol{\aleph}_{\mu}\right)$, and $h$ is isomorphic since no union of fewer than $\operatorname{ch}\left(\boldsymbol{\aleph}_{\mu}\right)$ sets $i\left(e^{\prime}\right)$ contains another $i\left(e^{\prime \prime}\right)$.

We conclude with remarks on four unsolved problems.

Problem 1. How many nonequivalent directed sets $D$ exist with $u(D) \leqq \boldsymbol{\aleph}_{1}$ ?

There are at least five: one trivial (zero type), three standard sets, and $\omega_{0} \times \omega_{1}$. These, but not more, can be distinguished by the values of $l(D)$, $u(D)$, and $\mathrm{bu}(D)$. Assuming the continuum hypothesis, $\pi\left(\boldsymbol{\aleph}_{0}\right)$ is a sixth. 5.2 shows that its principal bursting number is not $\boldsymbol{\aleph}_{0}$, whence (by 4.6) it is $\boldsymbol{\aleph}_{1}$. One must then distinguish $\pi\left(\boldsymbol{\aleph}_{0}\right)$ from $\omega_{0} \times \omega_{1}$. Of several simple proofs, perhaps this is the most informative. In $\pi\left(\boldsymbol{\aleph}_{0}\right)$, every unbounded subset contains a countable unbounded subset. Hence, as in the proof of 2.6, whenever $g: \pi\left(\boldsymbol{\aleph}_{0}\right) \rightarrow D \times \omega_{1}$ is a Tukey function, its first coordinate must already be a Tukey function.

The answer to Problem 1 may be $\boldsymbol{\aleph}_{2}$. Two particular sets whose position is unsettled are the filter of dense open sets in a Euclidean space and a 1product of $\boldsymbol{\aleph}_{1}$ copies of $\omega_{0}$. The former at least is not equivalent to $\Delta_{01}$.

Problem 2. How many nonequivalent ultrafilters exist on a set of power $\boldsymbol{\aleph}_{0}$ ? 
Between two (one trivial) and $\boldsymbol{\aleph}_{2}$ (if $(\mathrm{H})$ ).

We call a directed set $D$ directly irreducible if $B \times C \geqq D$ implies $B \geqq D$ or $C \geqq D$. Call $D$ completely irreducible if every set of directed sets $\left\{C_{\alpha}\right\}$ such that for all $\alpha, C_{\alpha} \geqq D$ is false, has an upper bound $B$ such that $B \geqq D$ is false. The obvious results are

Every cardinal set is directly irreducible. $\omega_{0}$ is completely irreducible. Hence the following questions seem reasonable.

Problem 3. Is every standard set directly irreducible?

Problem 4. Is every cardinal set completely irreducible?

Problem 3 is related to a problem of Fine and Gillman [2]. Rather than introduce their notation and state their conjecture (which implies direct irreducibility of $\Delta_{0_{\mu}}$ ), let us develop the relation for the first case of the problem. If $B \times C \geqq \Delta_{01}$, there is a Tukey function $g$ from the set $D=\Delta_{01}$ into $B \times C$. Regard $D$ as a discrete topological space; let $\beta D$ be its StoneČech compactification. Let $U \subset \beta D$ be the union of the closures of all subsets $S$ of $D$ such that $g(S)$ projects upon a bounded set in $B$. $U$ is an open set containing $D$. The sets with bounded images in $C$ similarly yield an open set $V$. For $g$ to be a Tukey function means $U \cap V=D$. If neither projection of $g(D)$ contains $\boldsymbol{\aleph}_{1}$ elements no $\boldsymbol{\aleph}_{0}$ of which are bounded, one has an answer to a question at the end of [2]; and the methods of [2] may be applicable.

On Problem 4 we actually have a negative result; by 3.9 , if $m$ is a $\rho$ number, $W(m)$ is not completely irreducible. The reader may restate the problem as he pleases. Negative results for any more cardinals would contribute to the theory of spectra; affirmative results for any more directed sets at all would be of interest.

\section{REFERENCES}

1. P. Erdös, L. Gillman and M. Henriksen, An isomorphism theorem for real-closed fields, Ann. of Math. (2) 61 (1955), 542-554

2. N. Fine and L. Gillman, Extension of continuous functions in $\beta N$, Bull. Amer. Math. Soc. 66 (1960), 376-381.

3. S. Ginsburg and J. Isbell, The category of cofinal types. I, Trans. Amer. Math. Soc. 116 (1965), 386-393.

4. F. Mahlo, Über lineare transfinite Mengen, Ber. Verh. Sächs. Ges. Wiss. Leipzig 63 (1911), 187-225.

5. E. Marczewski (E. Szpilrajn), Remarque sur les produits cartésiens d'espaces topologiques, C. R. Acad. Sci. URSS 31 (1941), 525-528.

6. J. Schmidt, Konfinalität, Z. Math. Logik 1 (1955), 271-303.

7. J. Tukey, Convergence and uniformity in topology, Annals of Mathematics Studies No. 2, Princeton, N. J., 1940.

INSTITUTE FOR ADVANCED STUDY,

Princeton, New Jersey

TULANE UNIVERSITY,

New Orleans, Louisiana 